\title{
MBSE als Datenbasis zur Unterstützung von Konfiguratoren und Digitalen Zwillingen modularer Produktfamilien
}

\section{MBSE as a databasis to support configuration systems and digital twins of modular product families}

\author{
Fabian Laukotka ${ }^{1 *}$, Florian Seiler ${ }^{1}$, Dieter Krause ${ }^{1}$ \\ ${ }^{1}$ Institute of Product Development and Mechanical Engineering Design, Hamburg University of Technology \\ ${ }^{*}$ Korrespondierender Autor: \\ Fabian Laukotka \\ Technische Universität Hamburg \\ Institut für Produktentwicklung und Konstruktionstechnik \\ Denickestraße 17, 21271 Hamburg \\ Telefon: 040428782325 \\ Mail: fabian.laukotka@tuhh.de
}

\begin{abstract}
Today more products are developed in the form of modular productfamilies with similar, but slightly different product variants. To improve the development process but also the use and lifespanning service of product families and individual products the usage of MBSE has been an uprising trend. Using system models implicate another benefit: The stored information can be accessed by applications and be used to improve the userinteraction. One example are product configurators allowing the user to create a product variant according to individual requirements.

This link to the product family's information can also be used in later lifecycle phases, as it enables an ongoing information flow about existing products into a digital representation, preparing the development and use of digital twins.
\end{abstract}

\section{Keywords}

MBSE, modular product families, product generator, digital twin 


\section{Motivation}

Im Rahmen der modularen Produktfamilienentwicklung gilt es, neben der reinen Entwicklung der Produktstruktur auch deren Anwendung und Pflege durch geeignete Hilfsmittel zu unterstützen. Solche Anwendung sind z.B. ein Produktkonfigurationssystem um den individuellen Kundenwünschen die möglichst optimale Produktvariante zuzuordnen. Ein weiterer Trend in der Produktentwicklung ist das Bestreben, die Modelle und Informationen über die Produktion hinaus mit den gefertigten Produkten verknüpft zu lassen. Durch die Kombination der Daten aus der Produktentwicklung und aktuellen IST-Daten einzelner Produkte entsteht eine spezifische digitale Repräsentation dieses Produkts; sein Digitaler Zwilling. Für solche Digitale Zwillinge gibt es eine Vielzahl von möglichen Anwendungsgebieten. Mit den Stichworten Wartungsoptimierungen durch Predictive Maintenance oder der Dokumentation des tatsächlichen Produktzustands für aufbauende Produktentwicklungen während der eigentlichen Nutzungsphase seien an dieser Stelle nur zwei Beispiele genannt. Für alle diese Anwendungsgebiete sowie die eigentliche Erstellung der Digitalen Zwillinge ist eine solide Grundstruktur der Daten und Informationen essentiell. Zudem müssen diese unterschiedlichsten Daten auch über Versionen hinweg nachvollziehbar und durchgehend konsistent sein. Für diese Anforderungen bietet das Model Based-Systems Engineering (MBSE) eine mögliche Lösung [1,2].

\section{Stand der Wissenschaft}

An dieser Stelle wird zunächst der Stand der Wissenschaft und Technik sowie das Verständnis einiger Begrifflichkeiten vorgestellt. Dies beginnt mit dem Verständnis von Produktfamilien und MBSE-Modellen beziehungsweise der Möglichkeit zur Interaktion mit diesen. Anschließend wird näher auf die beiden bereits genannten Trends des Produktkonfigurators sowie Digitaler Zwillinge eingegangen.

\subsubsection{Produktfamilien}

Traditionell beschäftigt sich die Produktentwicklung hauptsächlich mit einzelnen Produkten. Um Kunden ein, möglichst zu seinen Anforderungen passendes, Produkt anbieten zu können, setzen Firmen heutzutage jedoch zunehmend auf ganze Produktfamilien mit mehreren, teils nur geringfügig unterschiedlichen Produktenvarianten. Dadurch wird dem Kunden eine größere Vielfalt an Produktvarianten geboten, aus denen er die passendste aussuchen kann.

Vereinfacht dargestellt werden im Zuge der Planung und Entwicklung dieser Produktfamilien die Produktarchitektur sowie Charakteristika zunächst übergreifend beschrieben. Anschließend erfolgt die darauf aufbauende Beschreibung und Definition einzelner Produktvarianten, unter anderem über kundenrelevante Eigenschaften und Modulausprägungen [3]. Beide Prozesse werden begleitet von der Entstehung digitaler Dokumentationen und Modelle der Produktfamilie und insbesondere der einzelnen Produktvarianten. In der Produktion entstehen schließlich basierend auf diesen Modellen die fertigen Produkte.

\subsubsection{Interaktion mit MBSE-Modellen}

Hinsichtlich der kollaborativen Verwaltung großer und komplexer Produktarchitekturen erscheint die Verwendung klassischer Datenverarbeitungsmethoden nicht mehr sinnvoll. Vor allem die Verarbeitung verschiedenster Datentypen mit individuellen sowie variablen Objektverknüpfungen sind effektiv und dynamisch in eine Datenbasis zu integrieren. An diesem Ansatzpunkt bietet das Model-Based Systems Engineering (MBSE) eine mögliche Lösung für diese Herausforderung. Derzeit finden MBSE-Modelle bereits zunehmend mehr Verwendung in der Produktentwicklung. Dabei werden diese meist zur Modellierung oder 
Dokumentation verwendet. Vereinzelnd wird mit diesen Modellen auch über Schnittstellen in den Modellierungssoftwares, beispielsweise der JavaScript Macro Engine von Cameo Systems Modeller, interagiert. Dabei werden allerdings meist Abläufe oder Vorgänge in den Modellen ausgelöst, die gespeicherten Daten bleiben oftmals unverändert $[4,5,6,7]$.

$\mathrm{Da}$ mit zunehmender Verbreitung von MBSE-Modellen in verschiedenen Anwendungsfeldern auch die Anforderungen an solche Schnittstellen gewachsen sind, wird derzeit an der nächsten Generation der Modellierungssprache SysML gearbeitet. Mit SysML V2 steht derzeit die Entwicklung der nächsten Generation der wichtigen MBSESprache an, in der Schnittstellen, sogenannte APIs, explizit vorgesehen sind. Mit diesen Schnittstellen wird die Interaktion mit Modellen, so wie sie beispielhaft auch in dieser Arbeit vorgestellt wird, deutlich erleichtert [8].

\subsubsection{Konfiguratoren}

Da die endgültige Auswahl der jeweiligen Produktvariante für den Kunden auf Basis dessen individueller Gesamtheit an Kundenanforderungen geschehen muss, gilt es, stets die vollständige Produktarchitektur zu berücksichtigen. Insbesondere bei umfangreichen und komplexen Produktarchitekturen kann dies ohne geeignete Unterstützungsmöglichkeiten leicht zu Konsistenzproblemen bzw. zu inadäquaten und langwierigen Entscheidungsprozessen führen. Abhilfe kann hier ein entsprechend konzipierter Produktkonfigurator schaffen, dessen Logikstruktur den Inhalt und somit die Ontologie der Produktarchitektur mit deren Verknüpfungen zwischen Modulen und kundenrelevanten Eigenschaften widerspiegelt. Dementsprechend muss die Datenbasis eines solchen Konfigurationssystems in der Lage sein, qualitative Daten, wie Anforderungsverknüpfungen und kundenrelevante Eigenschaften etc., abzubilden. Herkömmliche PLM-Systeme haben ihre Stärke hauptsächlich in der Verwaltung von CAD-Daten, für den Einsatz als Datenmodellquelle eines Konfigurators hingegen erscheint der Einsatz eines flexiblen MBSE-Modells als sinnvoll. Dies wird umso deutlicher, wenn man die wichtigsten Anforderungen an Konfigurationssysteme betrachtet: Diese sind auf Basis eines vollständigen Informationssystems eine konsistente Produktkonfiguration sowie die Möglichkeit zur Plausibilitätsprüfung zu ermöglichen [9]. Liebisch zeigt auf, dass dies nur mit dem Einsatz einer geeigneten Modellierungssprache abgebildet werden kann, wie sie durch die Verwendung eines geeigneten MBSE-Tools zur Verfügung gestellt wird [10].

Auf Basis der relevanten Literatur lässt sich der Einsatz von MBSE als Erweiterung existierender PLM-Systeme, vor allem aber als Grundlage für ein flexibles, intelligentes Konfigurationssystem deutlich hervorheben [1]. Insbesondere sollen kundenrelevante Eigenschaften in die zu schaffende Modellstruktur als Basis eines Konfigurators integriert werden. Sendler et al. beschreiben die Verknüpfung von PLM und SysML als Grundlage für eine datentechnische Durchgängigkeit, indem nicht nur die Geometrie und Struktur eines Produktes gemanagt werden, sondern auch dessen Logik und Funktionen, welche als Anforderungen hinter diesem System stehen [11]. Vor allem die Vorwärts- und Rückwärtsintegration aller Systeme ist einer der größten Benefits, die mittels MBSE geschaffen werden.

\subsubsection{Digitale Zwillinge}

Der Begriff „Digitaler Zwilling“ erlebt derzeit geradezu einen Trend und ist Gegenstand vieler Berichte und Forschungsarbeiten. Dabei hängt das Verständnis, was genau darunter verstanden wird, sehr von dem individuellen Fokus und Anwendungsfeld der jeweiligen Arbeit ab. Jedes Anwendungsfeld hat dabei seine eigene Definition und Anforderungen an das, was unter einem Digitalen Zwilling verstanden wird. 
Eine Gemeinsamkeit stellt die Sichtweise dar, dass es sich um eine digitale Repräsentation eines Produkts oder Prozesses handelt. So beschreibt die Wissenschaftliche Gesellschaft für Produktentwicklung (WiGeP) einen Digitalen Zwilling als „eine digitale Repräsentation einer Produktinstanz [...] oder eines Produkt-Service-Systems“" [12]. Als Basis für einen Digitalen Zwilling wird dabei meist ein digitales Mastermodell genannt, das aus dem Entwicklungsprozess hervorgeht und anschließend mit Daten des spezifischen Produkts sowie weiteren Informationen, dem Digitalen Schatten, kombiniert wird (Abbildung 1). In der Literatur lässt sich der Begriff Digitaler Zwillinge jedoch auch im Kontext von z.B. der Fabrikplanung oder dem Management von Stromnetzen finden [13,14].

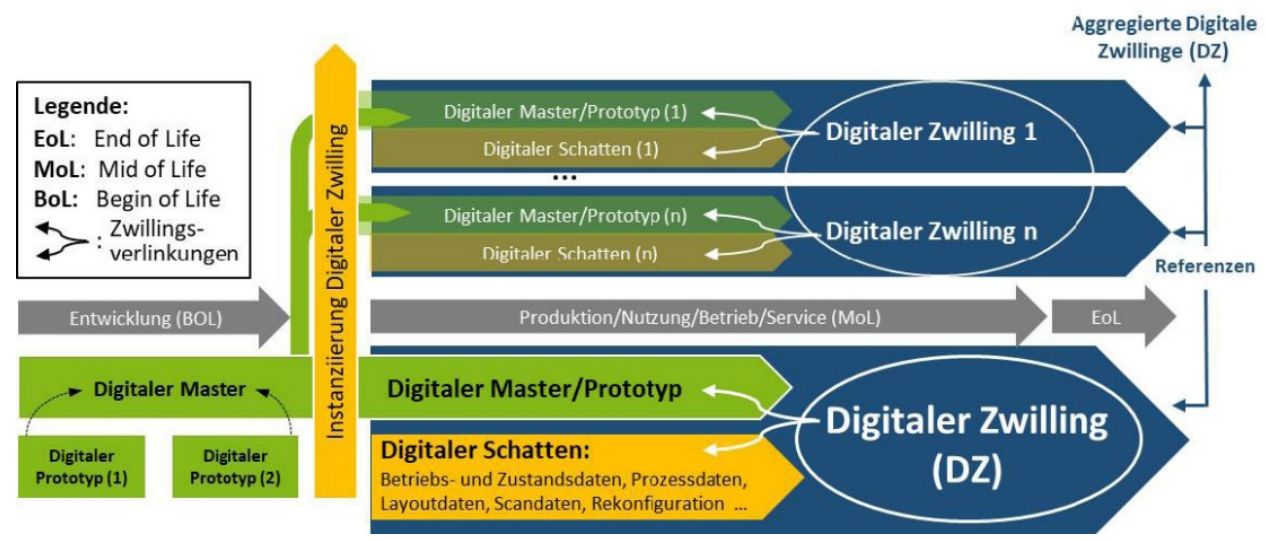

Abbildung 1: Definition des Digitalen Zwillings in Abhängigkeit von Produkt (unten) und Produktinstanz (oben) sowie Digitalem Master bzw. Prototyp und Digitalem Schatten [12]

Da die genaue Bedeutung hinter dem Begriff Digitaler Zwilling darüber hinaus nicht einheitlich ist, wird das Verständnis aus Sicht der Autoren mit Blick auf die Produktfamilienentwicklung im Folgenden näher beschrieben.

Im Kontext der Produktentwicklung wird sich bei den Forschungsarbeiten meist auf einzelne Produkte fokussiert. Wie in Kapitel 2.1.1 beschrieben stehen hinter einzelnen Produkten heutzutage jedoch oftmals ganze Produktfamilien mit den verschiedenen Produktvarianten. Der Ursprung von digitalen Abbildern der gefertigten Produkte liegt als nicht unbedingt im Mastermodell des Produkts, sondern in einer generischen Beschreibung der Produktfamilie, aus der anschließend die Mastermodelle der einzelnen Produktvarianten abgeleitet werden. Jedes spezifische, reale Produkt der einzelnen Produktvariante wird schließlich eine individuelle Instanz des Mastermodells zugeordnet.

Diese Modelle repräsentieren dabei den idealen Soll-Zustand, der durch Abweichungen beispielsweise in der Fertigung oder Veränderungen im Laufe des Produktlebens werden die einzelnen Produkte jedoch von diesem Ideal abweichen. Dadurch weisen die realen Produkte immer mehr Unterschiede zu den idealen Modellen aus der Produktentwicklung auf.

Wird dieses Mastermodell um die tatsächlichen Ist-Daten des spezifischen Produkts nach der Fertigung ergänzt, entsteht sein initiales digitales Abbild. Zu diesem Zeitpunkt ist das Abbild damit bereits mehr als nur das reine Mastermodell und geht über dessen digitale und ideale Beschreibung aus der Produktentwicklung mit den idealen Daten hinaus. Diese Abweichungen zum Mastermodell werden im Individuellen erfasst und schaffen damit die Grundlage für den Digitalen Zwilling.

Von einem Solchen kann jedoch erst gesprochen werden, wenn diese Daten im digitalen Abbild auch regelmäßig mit den tatsächlichen Ist-Daten aktualisiert werden. Dadurch entsteht die Möglichkeit für jedes Produkt Informationen über die Lebensphasen hinweg zur Verfügung zu stellen. Zu jedem einzelnen Produkt entstünde damit ein digitales Abbild, dass den genauen Zustand dieses spezifischen Produkts jederzeit repräsentiert; sein Digitaler Zwilling. Der Ursprung aller Digitalen Zwillinge aller Varianten der Produktfamilie liegt in der generischen 
Beschreibung des Produkts bzw. der Produktfamilie. Die einzelnen Digitalen Zwillinge unterscheiden sich dann hauptsächlich in der spezifischen Umsetzung oder Beschreibung. An dieser Stelle kann eine Analogie zu Klassen und Instanzen oder Objekten in der objektorientierten Softwareentwicklung gezogen werden [2].

\subsubsection{Fazit aus dem Stand der Technik / Forschungsfragen}

Zwei aktuelle Trends in der Produktentwicklung, die zunehmende kundenindividuelle Auswahl von Produktvarianten ermöglicht durch Produktfamilien, sowie die Ansätze digitale Repräsentationen der Produkte über die Produktentstehung hinaus zu verwenden, wurden in den vorherigen Teilkapiteln vorgestellt.

Nach Hermann ist die Konfiguration kundenspezifischer Produkte eine der Kernkompetenzen, die ein Unternehmen zu leisten hat. Hierbei wird ein intelligenter, dynamischer Produktkonfigurator als unumgänglich bezeichnet [15]. Konfiguratoren ermöglichen somit den Transfer des impliziten und expliziten Wissens aus der Modularisierung, indem diese Informationen intuitiv und dynamisch für den Kunden zur Verfügung gestellt werden.

Der zweite genannte Trend kann unter dem Begriff Digitaler Zwilling zusammengefasst werden. Dabei kann dieser in verschiedensten Gebieten Anwendung finden und die genaue Bedeutung dieses Begriffs je nach Forschungsarbeit und Anwendungsgebiet unterschiedlich. Daher wurde mit der Definition des Begriffes der WiGeP zunächst eine übergreifende Beschreibung und anschließend das genaue Verständnis der Autoren im Kontext dieser Arbeit beschrieben.

Während sich viele Forschungen mit einzelnen Anwendungsmöglichkeiten beschäftigen soll an dieser Stelle der Fokus auch auf die zugrundeliegende Struktur gelegt werden. Eine Herausforderung stellen dabei die unterschiedlichen Anforderungen an die Interaktionen mit der Datenbasis der verschiedenen Lebensphasen und deren verschiedenen Datentypen dar. Daher ist eine gemeinsame Datenbasis für alle Produkte aller Produktvarianten und für jede Lebensphase notwendig. Daraus ergeben sich vor allem folgende zwei Fragestellungen:

- Wie muss eine Datenstruktur sowie deren Schnittstellen aufgebaut sein, um die Entwicklung und Verwendung von Konfiguratoren für modularen Produktfamilien zu unterstützen?

- Wie kann das Wissen aus der Entwicklung von Produktkonfiguratoren für die Realisierung von Digitalen Zwillingen genutzt werden?

Wie bereits unter Abschnitt 2.1.3 beschrieben, ist hierzu ein konsistenter Datenstamm unbedingt nötig der u.a. mittels MBSE zur Verfügung gestellt werden kann. Dementsprechend erscheint die Anwendung vom MBSE als Backend zur Anknüpfung weiterer Systeme wie z.B. Konfiguratoren und im weiterführenden Sinne auch Digitale Zwillinge als sinnvoll.

Allerdings existiert in der aktuellen Version der verfügbaren SysML Tools keine direkte Möglichkeit zur direkten Datenextraktion aus MBSE-Modellen zur Anknüpfung weiterer Systeme (siehe 2.1.2). An dieser Stelle präsentiert der vorliegende Beitrag in den folgenden Kapiteln eine Möglichkeit zur Schnittstellenadaption am Beispiel des Produktkonfigurators. Anschließend wird die aktuelle Forschungsarbeit vorgestellt, diese für die Verwendung für Digitale Zwillinge zu adaptieren.

\section{Umsetzung und Anwendungsbeispiele}

Im folgenden Abschnitt wird der Einsatz von MBSE als Backend für Konfigurationssysteme modularer Produktfamilien sowie Digitaler Zwillinge beschrieben. Besonders hervorzuheben 
ist hierbei, dass MBSE in diesem Zusammenhang nicht als individuelle Methode mit einem spezifischen Modell verwendet wird, sondern über entsprechend entwickelte Schnittstellen als dynamische Datenbasis zum Einsatz kommt.

Um auf die bereits existierenden Forschungsergebnisse im Bereich der Datenbasis und Schnittstellen für ein MBSE-basiertes Konfigurationssystem aufsetzen zu können und analog dazu Digitale Zwillinge an die Modellumgebung anzubinden, erfolgt zunächst eine Beschreibung der MBSE-Umgebung als Konfigurationsbackend.

\subsection{Produktkonfiguratoren}

Zur Generierung und Pflege der immer umfassenderen, variantenreichen Produktarchitekturen werden modulare, dynamische Produktkonfiguratoren als zwingend nötig beschrieben [15]. Somit können explizite Kundenanforderungen optimal auf eine verfügbare Produktvariante abgebildet werden. Hierbei ist jedoch zu beachten, dass im Regelfall nicht die Gesamtheit der Kundenwünsche abgedeckt werden kann [10]. Da Kunden keine kundenrelevanten Eigenschaften kaufen, sondern sich auf deren Basis für variante Modulausprägungen entscheiden, kommt einem Konfigurator generell die Aufgabe zu, alle Informationen zwischen Kundenanforderungen und Stückliste zu verarbeiten. Hierbei kommen zwei interagierende Systeme zum Einsatz: das Frontend, welches die Nutzerschnittstelle darstellt und das Backend, das die Produktarchitektur dem Kunden zur Verfügung stellt.

Das MBSE eröffnet nun die Möglichkeit, Struktur- und Verhaltensinformationen sowie abstrahierte Verknüpfungen effizient nutzbar zu machen, um einzelne Modelle zu einem zusammenhängenden Metamodell zusammenzuführen. Hier bietet die Verwendung von SysML und MBSE einen entscheidenden Vorteil gegenüber alternativen Systemen, da eine durchgängige Abbildung von Logik, Struktur, Funktion und damit der Ontologie der Produktstruktur möglich wird [16]. Zur Verknüpfung von definierten Modulen mit spezifischen kundenrelevanten Eigenschaften muss die Datenbasis fähig sein, Anforderungsverknüpfungen und kundenrelevante Eigenschaften als qualitative Daten sowie deren quantitative Ausprägungen zugleich strukturiert und prozessierbar aufzunehmen. Die Notwendigkeit des Einsatzes von MBSE wird bei der Betrachtung der wichtigsten Anforderungen an Konfigurationssysteme umso deutlicher: Auf Basis eines vollständigen Informationssystems gilt es, eine konsistente Produktkonfiguration sowie die Möglichkeit zur Plausibilitätsprüfung zu ermöglichen. Dies kann nur mit dem Einsatz einer geeigneten Modellierungssprache abgebildet werden, wie sie durch die Verwendung eines geeigneten MBSE-Tools zur Verfügung gestellt wird [1].

Hierbei werden somit nicht nur die Geometrie und Struktur eines Produktes gemanagt, sondern auch dessen Logik und Funktion. Auf Basis dieser definierten Anforderungen gilt es, eine geeignete Datenstruktur auszuwählen, welche zugleich kundenrelevante Eigenschaften, Module, Komponenten und deren individuelle Verknüpfungen darstellen kann. Auf Basis der Analyse verschiedener methodischer Unterstützungstools bei der Entwicklung modularer Produktfamilien erscheint die Verwendung eines angepassten Netzplans aus Vertriebssicht auf Basis des Integrierten PKT-Ansatzes [3] als am geeignetsten.

Folgende Abbildung 2 zeigt die Implementierung eines beispielhaften Netzplans in der MBSE-Umgebung von Cameo Systems Modeler. Über die eindeutige Verknüpfung von kundenrelevanten Eigenschaften zu Modulen und somit zu den korrespondierenden Modulvarianten und deren einzelnen Komponenten kann die entwickelte Produktarchitektur vollständig modellbasiert abgebildet werden. Der für den vorliegenden Beitrag relevanteste Abschnitt betrifft die Definition, Darstellung und Modellierungen der individuellen Objektverknüpfungen, welche schlussendlich das Regelwert zur endgültigen Konfiguration von Produktvarianten darstellen. 


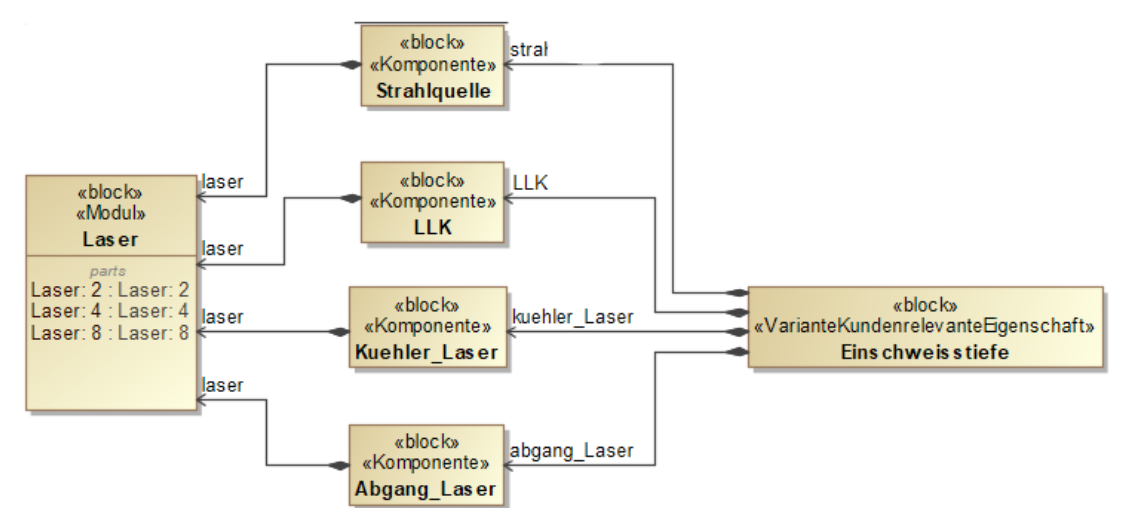

Abbildung 2: MBSE-Implementierung des konfigurationsfähig adaptierten Netzplans aus Vertriebssicht am Beispiel kundenindividuell gestalteter Laserschweißanlagen

Die Ausleitung dieser semantisch abgebildeten Produktstruktur sowie des Regelwerks über passende Schnittstellen ermöglicht anschließend eine konsistente Datenbasis für ein Konfigurationssystem. Somit kann für jede individuelle Zusammenstellung von Kundenanforderungen die passendste Produktvariante determiniert werden. Der Konfigurationsalgorithmus wurde auf Basis der genannten Anforderung generisch entwickelt, dass durch einen Zugriff auf Verknüpfungsmatrizen aus der MBSE-Umgebung diese Abhängigkeiten dynamisch während des Konfigurationsprozesses abgeleitet und angewendet werden. Abbildung 3 beschreibt den allgemeinen MBSE-basierten Konfigurationsprozess.

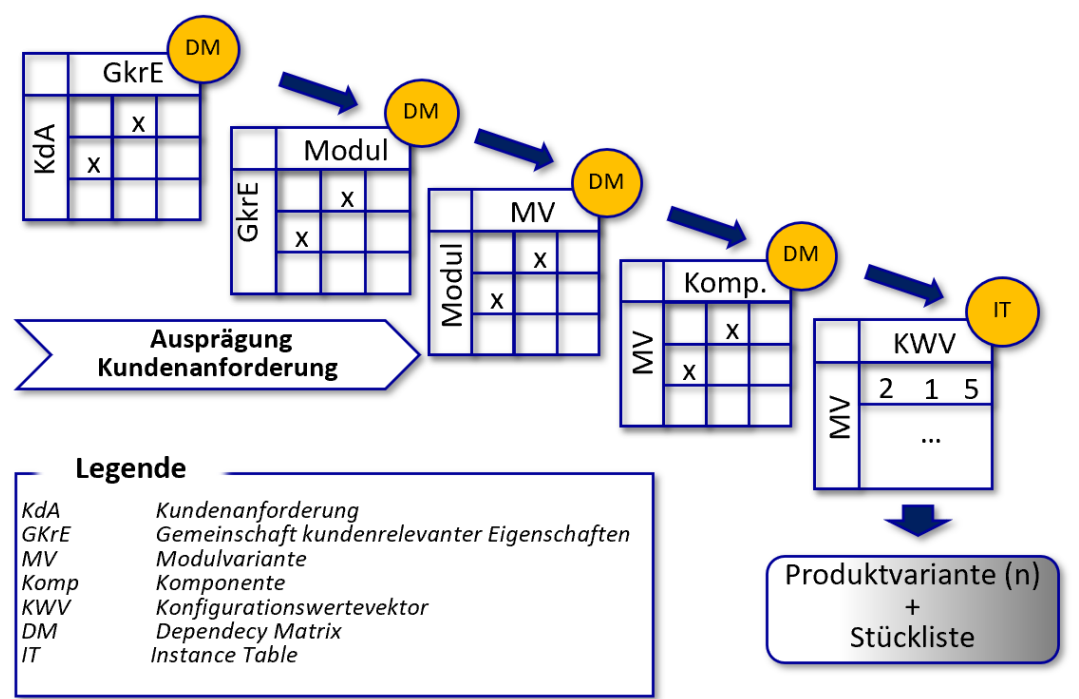

Abbildung 3: MBSE-basierter Konfigurationsprozess durch Schnittstellen-Matrizen

Die konfigurationsrelevanten Daten, wie z.B. Materialkosten der Einzelkomponenten, deren Lieferzeit oder der jeweilige Konstruktionsaufwand werden nach der Ermittlung der benötigten Modulvarianten den entsprechenden Komponenten zugeordnet. Da diese Daten den individuellen Parametern der Komponenten entsprechen, die bei der Zusammenstellung einer Modul- oder Produktvariante durch Aufsummieren einen Gesamtwert ergeben, werden diese Daten in Form eines Konfigurationswertevektors (KWV) attribuiert [17]. Diese Zuordnung sowie die Ausleitung der relevanten Daten über die Schnittstelle erfolgt durch die Verwendung eines entsprechend parametrisierten, rekursiv angewendeten Parameterdiagramm in der MBSE-Umgebung. Dieses sogenannte Rollup-Pattern ermöglicht eine schnelle, effiziente, konsistente und generische Datenzuordnung sowie Datenausleitung. Da durch den Konfigurationsprozess sämtliche Konfigurationswertevektoren zur Definition der bestimmten Produktvariante bekannt sind, kann eine "gültige“ Konfiguration somit auch mathematisch als 
Summe der KWV-Vektoren beschrieben werden. Diese vektorielle Darstellung einer Produktvariante eröffnet weitere zukünftige Möglichkeiten zur Bewertung und Analyse der jeweiligen Produktvariante oder der zugrundeliegenden modularen Produktstruktur.

\section{2. Übertragung auf die Grundlage für Digitale Zwillinge}

Die Verwendung von Systemmodellen und MBSE zur generischen Beschreibung von Produktfamilien ermöglicht nicht nur den zuvor beschriebenen Produktkonfigurator, sondern stellt zugleich auch eine mögliche Grundlage für die Realisierung von Digitalen Zwillingen dar. Denn die Digitalen Zwillinge verschiedener Instanzen desselben Produkts gehen alle auf die gleiche Basisstruktur zurück, haben allerdings individuelle Änderungen und Anpassungen durchlebt und unterscheiden sich daher. Hinzu kommen weitere Faktoren der Kommunalität und Varianz zwischen einzelnen Mastermodellen, wenn verschiedene Produkte einer Produktfamilie betrachtet werden. Die Mastermodelle der Produktvarianten weisen Gemeinsamkeiten auf, unterscheiden sich in unterschiedlichen Aspekten jedoch voneinander. Dadurch wird die Voraussetzung einer inkonsistenzfreien und möglichst vollständigen Datenbasis zu einer besonderen Herausforderung (vgl. Kapitel 2).

Die generische Beschreibung der Produktfamilie und darauf aufbauend der einzelnen Produktvarianten durch MBSE stellt dabei einen Ansatz dar, um diesen Anforderungen gerecht $\mathrm{zu}$ werden. Eine weitere Anforderung für die Nutzung Digitaler Zwillinge in den unterschiedlichen Lebensphasen eines Produkts ist eine Schnittstellte mit der verschiedene Anwendungen die Daten aus dem Digitalen Zwilling abrufen und anpassen, aber auch neue Daten einspeisen können [2].

Mit dem beschriebenen Produktkonfigurator mit MBSE-Basis wurde dieser Ansatz bereits in der Vergangenheit erprobt. Derzeit werden die dabei gewonnenen Erkenntnisse in aktuellen und kommenden Forschungsarbeiten auf das Konzept des Digitalen Zwillings übertragen. An dieser Stelle wird daher zunächst die generische Idee der Umsetzung dieser Adaption beschrieben. Bei genauerer Betrachtung lassen sich Synergieeffekte zwischen diesen beiden Themen feststellen. Sowohl der Produktkonfigurator als auch die Mastermodelle der Digitalen Zwillinge basieren auf dem gleichen Mastermodell, bestehend aus unter anderem BlockDefinitionsdiagrammen und Dependency-Matrices, der Produktfamilie. Vielmehr kann das Mastermodell eines spezifischen Produkts durch die Verwendung des Produktkonfigurators aus dem generischen Mastermodell der Produktfamilie abgeleitet werden. Dabei werden die Informationen und Zusammenhänge aus den Systemmodellen als eine Bauanleitung verwendet, um die Informationen und Daten der spezifischen Produktvariante in ihr eigenes Mastermodell zusammenzuführen. Mit diesem Mastermodell Im Zuge der Produktion dienen die Basis-Daten dieses spezifische Mastermodells der Produktvariante dann als Basis, die zusammen mit den Ist-Daten des tatsächlich gefertigten physischen Produkts den initialen Zustand des Digitalen Zwillings darstellen. Anpassungen durch die Benutzung oder Veränderungen im Laufe des Lebens des physischen Produkts müssen schließlich regelmäßig in den Digitalen Zwilling zurückgespielt werden können.

Abbildung 4 beschreibt die Anordnung und Verknüpfung von Produktkonfiguratoren und Digitalen Zwillingen in den jeweiligen Lebensphasen der Produktfamilie. Für die Realisierung ist eine gemeinsame, übergreifende und vor allem konsistente Datenbasis essenziell. Die Definition und Darstellung der WiGeP (vgl. Kapitel 2.1.4, Abbildung 1) wird aufgegriffen und um die zuvor beschriebenen Prozesse bei der Betrachtung von Produktfamilien, Produktvarianten und Produktkonfiguratoren erweitert. Die geschwungenen unteren Enden der Darstellung von Produktfamilie und ihrem Mastermodell sowie dem Mastermodell der Produktvariante deuten dabei an, dass es zu jeder Produktvariante eine Vielzahl an individuellen produzierten Instanzen geben kann und innerhalb der Produktfamilie ebenfalls eine Vielzahl an Produktvarianten vorkommen kann. Die Darstellung zeigt somit nur den Ausschnitt einer Produktinstanz einer Produktvariante der Produktfamilie. 


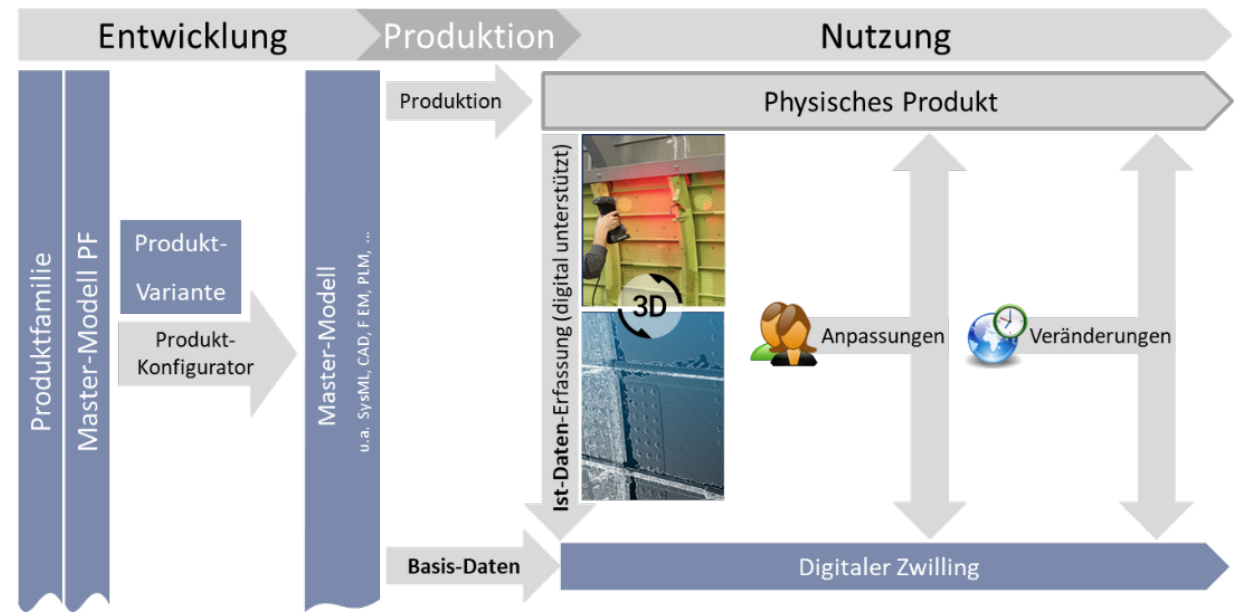

Abbildung 4: Der Produktkonfigurator und Digitale Zwillinge im Anfang des Lebenszyklus von Produktfamilien

Sowohl der Produktkonfigurator als auch die Basis-Daten der Digitalen Zwillinge gehen bei dieser Umsetzung auf eine generische Datenbasis zurück. In Bezug auf die Umsetzung unterscheiden sich die beiden Anwendungen jedoch: Während beim Produktkonfigurator das Meta-Modell einmalig zur Auswahl der benötigten CAD-Daten verwendet wird, werden beim Digitalen Zwilling die CAD-Daten und weitere Informationen über die Struktur des MetaModells verwaltet und regelmäßig für die Aktualisierung des Zwillings verwendet. In beiden Fällen, dient das Meta-Modell als eine Art Bauplan des spezifischen Produkts und den dazu gehörenden Informationen. Je nach betrachteter Lebensphase können dies dabei unterschiedliche Informationen und Datentypen sein. Durch die generische Struktur lassen sich bei einem bestehenden Datensatz auch Auswertungen und Analysen der Daten über die ganze Produktfamilie hinweg durchführen. Diese gesamtheitliche Betrachtung des Produktlebenszyklus ist der vielversprechendste Weg für die Entwicklung, Umsetzung und Verwendung von Digitalen Zwillingen. Dieser Prozess kann durch MBSE unterstützt werden.

Während der Produktkonfigurator bereits umgesetzt wurde, ist die hier beschriebene Adaption für die Realisierung Digitaler Zwillinge Gegenstand aktueller Forschung. Aus diesem Grund ist eine mögliche Umsetzung basierend auf den Erkenntnissen des Konfigurators an dieser Stelle lediglich generisch beschrieben.

\section{Diskussion und Ausblick}

Zur Anbindung eines Produktkonfigurators unter Verwendung des in einer MBSEUmgebung abgebildeten modularen Baukastens gilt es, primär die Transformation der Kundenperspektive und somit der Kundenanforderungen in die Unternehmensperspektive (kundenrelevante Eigenschaften) zu bewältigen.

Da die zur Verfügung stehenden Softwarepakete zur MBSE-Modellierung auf die reine Implementierung von modellbasierten Strukturen ausgerichtet sind, müssen anknüpfende Systeme wie z.B. Produktkonfiguratoren oder Digitale Zwillinge über eine Schnittstelle auf die Struktur zugreifen können. Hier stellen sich dieselben Anforderungen an die Schnittstelle wie an die initiale Modellierung des Datenmodells, wie z.B. die Verarbeitung verschiedener Datentypen sowie deren Verknüpfungen, eine vollständige und gleichzeitig ressourceneffiziente Datenbereitstellung sowie eine Wahrung der Konsistenz.

Zur Anbindung eines Produktkonfigurators unter Verwendung des in einer MBSEUmgebung abgebildeten modularen Baukastens gilt es, primär die Transformation der Kundenperspektive in die Unternehmensperspektive zu bewältigen [10]. Der Konfigurationsalgorithmus hierfür wurde auf Basis der oben genannten Anforderung derart generisch entwickelt, dass durch einen Zugriff auf exportierte Verknüpfungsmatrizen aus der 
MBSE-Umgebung diese Abhängigkeiten dynamisch während des Konfigurationsprozesses abgeleitet und angewendet werden. Die konfigurationsrelevanten Daten werden nach der Ermittlung der benötigten Modulvarianten den entsprechenden Komponenten zugeordnet. Da diese Daten den individuellen Parametern der Komponenten entsprechen, die bei der Zusammenstellung einer Modul- oder Produktvariante durch entsprechendes Aufsummieren einen Gesamtwert ergeben, werden diese Daten in Form eines Konfigurationswertevektors (KWV) attribuiert. Diese Zuordnung sowie die Ausleitung der relevanten Daten über die Schnittstelle erfolgt durch die Verwendung eines entsprechend parametrisierten, rekursiv angewendeten Parameterdiagramms in der MBSE-Umgebung [1].

Aufbauend auf der Schnittstelle für den Produktkonfigurator wurden erste Schritte zur Übertragung des Know-Hows auf Ansätze zur Realisierung digitaler Zwillinge von Produktfamilien unternommen [2]. Weitere Arbeiten auf diesem Gebiet sind Gegenstand aktuell beginnender Forschungsprojekte. Anwendung fände dies beispielsweise in der Flugzeug-Kabinenentwicklung, genauer dem Retro-Fit alter Flugzeuge mit neuen Kabinen, bei denen genauen Informationen über den Ist-Zustand einzelner Maschinen notwendig sind [18].

\section{Literaturverzeichnis}

[1] Seiler, Florian; Schwede, Lea-Nadine; Krause, Dieter: „MBSE-basierte Produktkonfiguratoren zur Analyse der Modularisierung bei der Entwicklung modularer Baukasten-systeme“. Entwerfen Entwickeln Erleben in Produktenwicklung und Design, Band 2. 27. - 28. Juni 2019, Dresden

[2] Laukotka, Fabian. et al.: „Lebensphasenübergreifende Nutzung Digitaler Zwillinge - Modellbasierte Produktfamilienentwicklung am Beispiel der Flugzeugkabine“, in ZWF Zeitschrift für wirtschaftlichen Fabrikbetrieb: Vol. 115, No. special, pp. 101-104, DOI: 10.3139/104.112332

[3] Krause, Dieter; Gebhardt, Nicolas: „Methodische Entwicklung modularer Produktfamilien - Hohe Produktvielfalt beherrschbar entwickeln“, Springer Verlag, 2018, DOI: 10.1007/978-3-662-53040-5

[4] Bursac, Nikola (2016): „Model Based Systems Engineering zur Unterstützung der Baukastenentwicklung im Kontext der Frühen Phase der Produktgenerationsentwicklung“, Dissertation. Stolzenberger, Leimen.

[5] Schwede, Lea-Nadine, et al.: „Consistent Modelling of the Impact Model of Modular Product Structures with Linking Boundary Conditions in SysML". in "Proceedings of the 22nd International Conference on Engineering Design (ICED19)", Delft, 2019. DOI: 10.1017/dsi.2019.367

[6] Hanna, Michael, Schwenke, Johann, Krause, Dieter: „Inconsistency management for product families with many variants through a model-based approach in modular lightweight design" in "Proceedings of the Design Society: DESIGN Conference 2020" pp. 917-926, DOI: 10.1017/dsd.2020.309

[7] Küchenhof, Jan et al.: „From Visualizations to Matrices - Methodical support for New Development of Modular Product Families", 21th International DSM Conference, DSM 2019, Monterey, USA, 2019.

[8] Weilkiens, Tim: „NextGenSysML“, November 2018, Online-Artikel, abgerufen von: www.oose.de/blogpost/next-generation-modeling-teil-0-sysml-v2-und-sysml-api-services-einfuehrung,

[9] Göbel, Andreas: „Internationalisierung in Produktkonfiguratoren - Anforderungen und Konzepte für die Datenhaltung“. Diplomarbeit, 2009, Jena

[10] Liebisch, Matthias: „Aspektorientierte Datenhaltung in Produktkonfiguratoren - Anforderungen, Konzepte und Realisierung“. Dissertation, 2014, Jena

[11] Sendler, Ulrich; Weilkiens, Tim: „Modellbasierte Systementwicklung. Was Sie schon immer über MBSE, PLM und Industrie 4.0 wissen sollten" (I). Tag des Systems Engineering 2013.

[12] WiGeP-Positionspapier: „Digitaler Zwilling“. ZWF Zeitschrift für wirtschaftlichen Fabrikbetrieb: Vol. 115, No. special, pp. 47-50, DOI: 10.3139/104.112311

[13] ZWF Zeitschrift für wirtschaftlichen Fabrikbetrieb: Vol. 115, 2020, No. special, pp. 1-2, DOI: 10.3139/104.020DZ1

[14] Doleski, Oliver D.: „Realisierung Utility 4.0: Praxis der digitalen Energiewirtschaft“, Springer Verlag, 2020, DOI: 10.1007/978-3-658-25332-5

[15] Hermann, Marc-Oliver; Michler, Johannes; Schönthaler, Frank: „Wo Kundenwünsche auf technische und wirtschaftliche Notwendigkeiten treffen“. Business News 03/2013, abgerufen von http://bs.doag.org

[16] Haskins, Cecilia et al.: Incose - Systems Engineering Handbook: A Guide for System Life Cycle Processes and Activities, 2011, San Diego

[17] Seiler, Florian; Krause, Dieter: „A Multi-Dimensional Configuration Algorithm for Modular Product Architectures". in Proceedings of the Design Society: DESIGN Conference, pp. 2405-2414, Dubrovnik, 2020

[18] Laukotka, Fabian; Oltmann, Jan; Krause, Dieter: „A Digitized Approach to Reduce Assembly Conflicts During Aircraft Cabin Conversions" 30. DfX-Symposium, Hamburg 2019, DOI: 10.35199/dfx2019.22 